\title{
From Stylized to Quantitative Spatial Models of Cities
}

Sonya Ravindranath Waddell and Pierre-Daniel Sarte

\section{INTRODUCTION}

Understanding how and why factors of production locate within and around urban areas has been compelling social scientists for at least 150 years. Within mainstream economics, urban economists have been developing modern theories of city systems at least since the 1960s. However, modeling spatial interactions is highly complex, and, therefore, the theoretical literature on economic geography has necessarily focused on stylized settings. For example, a model may have a central business district - where firms are assumed to be located - surrounded by a symmetric circle or on a symmetric line. As the population grows, the scarcity of land prevents consumers (who are also workers) from all settling close to the center, so people move out to where commuting costs are higher but housing costs are lower.

In the models of new economic geography (NEG), urban economists have incorporated advances developed in industrial organization, international trade, and economic growth to remove technical barriers to modeling cities. The field of NEG was initiated primarily by three authors: Fujita (1988), Krugman (1991), and Venables (1996), who all use general equilibrium models with some version of monopolistic competition. The NEG models have been useful in helping to pin down preferences, technology, and endowments and have provided

- We thank Daniel Schwam and Daniel Ober-Reynolds for helpful research assistance. The views expressed herein are those of the authors and do not necessarily represent the views of the Federal Reserve Bank of Richmond or the Federal Reserve System. We thank Urvi Neelakantan, Santiago Pinto, and John Weinberg for helpful comments and suggestions. 
some fundamental theoretical explanation for the uneven distribution of economic activity, for multiple equilibria in location choices, and for a small (possibly temporary) asymmetric shock across sites to generate a large permanent imbalance in the distribution of economic activities. However, these models have also imposed structure that is not necessarily evident in the data, and the limitation of the analysis to stylized spatial settings has not enabled an empirical literature that could directly corroborate the theory. In other words, the stylized models have only guided empirical estimation in a way that is divorced from the structure of those models, resulting in empirical research that has been devoid of strong structural interpretations.

More recently, the introduction of quantitative models of international trade (in particular Eaton and Kortum [2002]) have served to develop a framework that connects closely to the observed data. This research does not aim to provide a fundamental explanation for the agglomeration of economic activity but instead aims to provide an empirically relevant quantitative model. This article describes the progression from a simple canonical model of NEG to its counterpart in the quantitative spatial framework. Section 2 engages the literature to develop and understand the progression from the stylized models of the NEG literature to the quantitative spatial models. Section 3 walks through a version of the stylized model, with a linear monocentric city. Section 4 introduces its counterpart as a quantitative spatial model as was laid out in Redding and Rossi-Hansberg (forthcoming). Section 5 provides an example of how the spatial model can be matched to detailed microdata that describe actual interactions in the city. Section 6 concludes.

\section{LITERATURE REVIEW}

The standard monocentric model of cities came out of a history of work to model spatial allocations. The prototype for understanding how factors of production distribute themselves across land, and how prices govern that distribution, was developed by Johann Heinrich von Thünen in the mid-nineteenth century to describe the pattern of agricultural activities in preindustrial Germany. Von Thünen's model includes an exogenously located marketplace in which all transactions regarding final goods must occur and the differences in land rent and use are determined predominantly by transport costs (Fujita and Thisse 2002). The von Thünen model was both formalized mathematically and enhanced in the second half of the twentieth century-including the formalization of bid-rent curves by William Alonso in his basic urban land model. This basic urban model includes a monocentric city 
Waddell \& Sarte: From Stylized to Quantitative Spatial Models of Cities171

with a center, home to the central business district (CBD), where all jobs are located. The space surrounding the CBD is assumed to be homogenous with only one spatial characteristic: its distance to the CBD. Both the work of von Thünen and that of Alonso depended upon the monocentricity of production activities - i.e., the models rely on one CBD (or market) with surrounding land used for residential (or agricultural) purposes.

Although many early models assumed the existence of the CBD, later work formalized mechanisms for the agglomeration forces that create concentrations of economic activity. The models of NEG, as summarized in Fujita et al. (1999), Fujita and Thisse (2002), and Ottaviano and Thisse (2004), create the framework to explain the imbalance in the distribution of economic activity and better understand how a small shock can generate that imbalance. These NEG models went a long way toward overcoming the fundamental problem that kept economic geography and location theory at the periphery of mainstream economic theory for so long: regional specialization and trade cannot arise in the competitive equilibrium of an economy with homogenous space. This spatial impossibility theorem is discussed more thoroughly in Ottaviano and Thisse (2004) and articulated mathematically in Fujita and Thisse (2002).

Important ideas underlie the development of the NEG models. These ideas (as described in Ottaviano and Thisse [2004]) include that the distribution of economic activity is the outcome of a trade-off between various forms of increasing returns and different mobility costs; price competition, high transport costs, and land use foster the dispersion of production and consumption, and, therefore, firms are likely to cluster in large metropolitan areas when they sell differentiated products and transport costs are low. Cities provide a wide array of final goods and specialized labor markets that make them attractive to consumers/workers, and agglomeration is the outcome of cumulative processes involving both the supply and demand sides. The contribution of NEG was to link those ideas together in a general equilibrium framework with imperfect competition. Some of the earliest work in NEG came from Krugman (1991), who developed a model that showed that the emergence of an industrialized "core" and an agricultural "periphery" pattern depends on transportation costs, economies of scale, and the share of manufacturing in national income (i.e., in consumption expenditures). More specifically, in his model, lower transportation costs, a higher manufacturing share, or stronger economies of scale will result in the concentration of manufacturing in the region that gets a head start compared to other regions. Venables (1996) wrote a model where imperfect competition and transport costs create 
forward and backward linkages between industries in different locations. He finds that even without labor mobility, agglomeration can be generated through the location decisions of firms in industries that are linked through an input-output structure. The models above develop an argument for agglomeration into a single center of activity. However, other NEG models, most notably Fujita and Ogawa (1982) and Lucas and Rossi-Hansberg (2002), introduced nonmonocentric models where businesses and housing can be located anywhere in the city. The latter models constitute a first step toward building frameworks that more accurately capture the heterogeneity in economic activity across space.

Unfortunately, although the theoretical work on NEG has been relatively rich, the empirical research has been comparatively less rich; establishing causality and controlling for confounding factors has proved challenging in the empirical realm. One challenge, as articulated by Redding and Rossi-Hansberg (forthcoming), is that the complexity of the theoretical models has limited the analysis to stylized spatial settings, such as a few locations, a circle, or a line, and the resulting empirical research has been primarily reduced form in nature. As a result, it is difficult to provide a structural interpretation of the estimated coefficients, and the empirical models cannot either withstand the Lucas critique (coefficients might change with different policy interventions) or necessarily generalize to more realistic environments.

Empirical work, such as the spatial model laid out in Section 4, has been instructed by another field of economics. Developments in the international trade literature have offered mechanisms for better modeling the distribution of economic activity across urban areas. Eaton and Kortum (2002) developed a model of international trade that captures both the comparative advantage that encourages trade and the geographic barriers that inhibit it (e.g., transport costs, tariffs and quotas, challenges negotiating trade deals, etc.). They use the model to solve for the the world trading equilibrium and examine its response to policies.

This framework from the trade literature - combined with the availability of increasingly more granular data - enabled the emergence of new quantitative spatial models in urban economics in which one can carry out general equilibrium counterfactual policy exercises. In addition to offering methodological insights and a mechanism for policy analysis, these quantitative spatial models have made substantive contributions that borrow from, and contribute to, the theoretical literature. For example, Redding and Sturm (2008) provide evidence for a causal relationship between market access and the spatial distribution of economic activity. They show that the division of Germany after 
Waddell \& Sarte: From Stylized to Quantitative Spatial Models of Cities173

World War II led to a sharp decline in population growth in West German cities close to the new border relative to other West German cities and that this decline was more pronounced for small cities than for large cities. As another example, models such as those developed in Ahlfeldt et al. (2015) and Monte et al. (2016), allow for heterogenous gradients of economic activity within cities that can be matched directly to microdata and that can only be approximated in models such as Fujita and Ogawa (1982) and Lucas and Rossi-Hansberg (2002).

The next section walks through a canonical monocentric urban model and highlights key features that made that model attractive for thinking about the distribution of economic activity across space. In particular, this urban model allows many of a city's features to be endogenous, including its size, population, employment, wages, and commercial land rents. In addition, at different locations within the city, residential population, residential prices, and the consumption of housing services can also be endogenous. In this model, as in the average city, production is concentrated at the center, where the CBD is located, rent gradients decline with distance from the CBD, and population density tends to decrease away from the city center.

\section{A STYLIZED MODEL OF CITIES}

We consider a linear monocentric city with locations defined on the interval $[-B, B]$, where $\ell$ denotes the distance from the city center. Each location $\ell$ is endowed with one unit of land available either for residential housing or production. This analysis focuses on residential localization decisions, i.e., the decisions of households rather than firms.

\section{The Central Business District}

All production takes place at the city center, $\ell=0$, which defines the CBD. Production per unit of land is given by

$$
Y=A(L) L^{\beta},
$$

where $L$ denotes labor input and $A(L)$ denotes a production externality. For simplicity, let $A(L)=A L^{\alpha}, \alpha<1-\beta<1$, and denote the wage paid to workers by $w$. This condition ensures that labor demand, $L$, is decreasing in the wage, $w$. There exists a unit mass of firms (assuming firms are small and do not internalize the externality) where the representative firm solves

$$
\max _{L} A(L) L^{\beta}-w L
$$


It follows that

$$
\beta A(L) L^{\beta-1}=w \Leftrightarrow L=\left(\frac{A \beta}{w}\right)^{\frac{1}{1-\alpha-\beta}} .
$$

We assume a competitive market with free entry so that in equilibrium firms obtain zero profits. Therefore, the commercial bid rent faced by firms in the business district is

$$
q^{b}=(1-\beta) A^{\frac{1}{1-\alpha-\beta}}\left(\frac{\beta}{w}\right)^{\frac{\alpha+\beta}{1-\alpha-\beta}} .
$$

\section{Residential Areas}

Workers live in the city at different locations, $\ell \in[-B, B] \backslash\{0\}$, and commute to the city center. Workers who reside at $\ell$ consume goods, $c(\ell)$, housing services, $h(\ell)$, and experience a commuting cost, $\kappa(\ell) \in$ $[1, \infty)$, that reduces the utility derived from housing and increases with distance from the CBD. In particular, the utility of a worker commuting from location $\ell$ to the CBD is given by $s\left(\frac{c(\ell)}{\gamma}\right)^{\gamma}\left(\frac{h(\ell)}{(1-\gamma) \kappa(\ell)}\right)^{1-\gamma}$, where $\gamma \in(0,1)$ and $s$ is a service amenity conferred by the city. This approach to modeling commuting costs departs somewhat from the more traditional approach of assuming that disposable income (thus consumption of housing and nonhousing goods) declines with distance from the CBD. In this case, similar to Ahlfeldt et al. (2015), commuting costs enter the utility function multiplicatively, which, as they note, is isomorphic to a formulation in terms of a reduction in effective units of labor. Commuting costs are then ultimately proportional to wages in the indirect utility function.

Conditional on living at location $\ell$, a worker then solves

$$
\begin{aligned}
u(\ell) & =\max _{c(\ell), h(\ell)} s\left(\frac{c(\ell)}{\gamma}\right)^{\gamma}\left(\frac{h(\ell)}{(1-\gamma) \kappa(\ell)}\right)^{1-\gamma}, \\
\gamma & \in(0,1)
\end{aligned}
$$

subject to $c(\ell)+q^{r}(\ell) h(\ell)=w$,

where $q^{r}(\ell)$ is the price of a unit of residential housing services at location $\ell$. Hence, we have that

$$
\begin{aligned}
c(\ell) & =\gamma w \\
h(\ell) & =\frac{(1-\gamma) w}{q^{r}(\ell)},
\end{aligned}
$$


Waddell \& Sarte: From Stylized to Quantitative Spatial Models of Cities175

and

$$
\begin{aligned}
u(\ell) & =s[w]^{\gamma}\left[\frac{w}{\kappa(\ell) q^{r}(\ell)}\right]^{1-\gamma} \\
& =s w\left[\kappa(\ell) q^{r}(\ell)\right]^{\gamma-1}
\end{aligned}
$$

\section{The Residential Market}

Let $\bar{u}$ denote the utility available to workers from residing in alternative cities. To the extent that workers can move to or from another city and are free to reside at any location within the city, it must be the case that in equilibrium $u(\ell)=\bar{u} \forall \ell \in[-B, B]$. Therefore, from equation (6), we have that, for any location $\ell$,

$$
s w\left[\kappa(\ell) q^{r}(\ell)\right]^{\gamma-1}=s w\left[\kappa(B) q_{B}^{r}\right]^{\gamma-1},
$$

where $q_{B}^{r}$ is the price of land at the boundary of the city defined by the opportunity cost of land at that location, such as an agricultural land rent. Rewriting equation (7) gives residential land rents at different locations within the city,

$$
q^{r}(\ell)=\frac{\kappa(B)}{\kappa(\ell)} q_{B}^{r}
$$

where $\frac{\kappa(B)}{\kappa(\ell)} \geq 1 \forall \ell \in[-B, B]$, since $\kappa(\ell)$ increases with distance from the city center. Thus, residential land rents are highest near the CBD and decrease toward the boundaries of the city as commuting becomes more expensive. However, as seen from equation (5), total housing expenditures in this framework are constant across all locations in the city since $q^{r}(\ell) h(\ell)=(1-\gamma) w$, where $(1-\gamma)$ then represents the income share of housing expenditures.

Recall that each location $\ell \in[-B, B]$ is endowed with one unit of land available for housing. Let $R(\ell)$ denote the residential population living at $\ell$. We assume that all available land in the city is fully developed and used by residents. Then, equilibrium in the housing sector requires that

$$
R(\ell) h(\ell)=1 \text {. }
$$


In addition, the residential population living at different locations $\ell$ in the city must sum up to the supply of labor working in the CBD,

$$
\begin{aligned}
\int_{-B}^{B} R(\ell) d \ell & =L \\
& \Rightarrow \int_{-B}^{B} \frac{1}{h(\ell)} d \ell=L
\end{aligned}
$$

\section{Solving for the City Equilibrium}

We now describe the city equilibrium, first solving for equilibrium wages as a function of the model parameters, from which all other city allocations immediately follow.

Given equations (5) and (8), equation (10) becomes

$$
\int_{-B}^{B} \frac{1}{h(\ell)} d \ell=\int_{-B}^{B} \frac{q^{r}(\ell)}{(1-\gamma) w} d \ell=\frac{\kappa(B) q_{B}^{r}}{(1-\gamma) w} \int_{-B}^{B} \frac{1}{\kappa(\ell)} d \ell=L,
$$

which defines the boundaries of the city, $B(L, w)$, as a function of its population and wages given the model's parameters.

Consider for instance the simple symmetric case where $\kappa(\ell)=e^{\kappa|\ell|}$ so that $\kappa(0)=1$ and $\kappa(B)=e^{\kappa B}>1$. Then, $\int_{-B}^{B} \frac{1}{\kappa(\ell)} d \ell$ gives

$$
\int_{-B}^{B} \frac{1}{\kappa(\ell)} d \ell=\int_{-B}^{B} e^{-\kappa|\ell|} d \ell=2 \int_{0}^{B} e^{-\kappa \ell} d \ell=2\left(\left.\frac{-e^{-\kappa \ell}}{\kappa}\right|_{0} ^{B}\right)=\frac{2}{\kappa}\left(1-e^{-\kappa B}\right)
$$

so that equation (11) becomes

$$
\begin{aligned}
\frac{\frac{2}{\kappa} e^{\kappa B} q_{B}^{r}\left(1-e^{-\kappa B}\right)}{(1-\gamma) w} & =\frac{\frac{2}{\kappa} q_{B}^{r}\left(e^{\kappa B}-1\right)}{(1-\gamma) w}=L \\
& \Rightarrow e^{\kappa B}=1+\frac{\kappa(1-\gamma) w L}{2 q_{B}^{r}}
\end{aligned}
$$

Using the labor demand equation in equation (2), conditional on the model parameters, the boundaries of the city may then alternatively 
Waddell \& Sarte: From Stylized to Quantitative Spatial Models of Cities177

be expressed in terms of wages only,

$$
\begin{aligned}
e^{\kappa B} & =1+\frac{\kappa(1-\gamma) w}{2 q_{B}^{r}}\left(\frac{A \beta}{w}\right)^{\frac{1}{1-\alpha-\beta}} \\
& =1+\frac{\kappa(1-\gamma)(A \beta)^{\frac{1}{1-\alpha-\beta}} w^{\frac{-\alpha-\beta}{1-\alpha-\beta}}}{2 q_{B}^{r}} .
\end{aligned}
$$

Using this last expression, we can solve for equilibrium wages in the city as a function of the model parameters only. Specifically, note that residents' utility at the boundary is given by

$$
\bar{u}=s w\left[q_{B}^{r}+\frac{\kappa(1-\gamma)(A \beta)^{\frac{1}{1-\alpha-\beta}} w^{\frac{-\alpha-\beta}{1-\alpha-\beta}}}{2}\right]^{\gamma-1},
$$

which defines $w^{*}=w\left(s, \kappa, \gamma, A, \alpha, \beta, q_{B}^{r}, \bar{u}\right)$.

Proposition 1: There exists a unique $w^{*}$ that solves equation (12).

Proof: Define $f(w)=s w\left[q_{B}^{r}+\frac{\kappa(1-\gamma)(A \beta)^{\frac{1}{1-\alpha-\beta}} w^{\frac{-\alpha-\beta}{1-\alpha-\beta}}}{2}\right]^{\gamma-1}$. Then $\lim _{w \rightarrow 0} f(w)=0, \lim _{w \rightarrow \infty} f(w)=\infty$, and, since $f(w)$ is continuous in $w$, there exists $w^{*}$ such that $\bar{u}=f\left(w^{*}\right)$. Moreover, since $f(w)$ is strictly increasing in $w, w^{*}$ is unique.

Given $w^{*}$, all other allocations in the city then immediately follow. In particular, as mentioned in the proposition, given parameter restrictions, the RHS of equation (12) is increasing in $w$ so that $w^{*}$ then increases with $\bar{u}$. Thus, as the reservation utility from living elsewhere, $\bar{u}$, increases, the city population, $L^{*}=\left(\frac{A \beta}{w^{*}}\right)^{\frac{1}{1-\alpha-\beta}}$, falls as residents leave the city, and its boundaries, $B^{*}=\frac{1}{\kappa} \log \left(1+\frac{\kappa(1-\gamma)(A \beta)^{\frac{1}{1-\alpha-\beta}} w^{\frac{-\alpha-\beta}{1-\alpha-\beta}}}{2 q_{B}^{r}}\right)$, shrink.

The stylized model described above is rich enough to allow for many of a city's features to be endogenous, including its size, population, employment, wages, and commercial land rents. In addition, at different locations within the city, residential population, residential prices, and the consumption of housing services can also be endogenous. These allocations are such that there exists a very direct link between commuting costs to the CBD and residential prices. Specifically, taking equation (8) and using the functional form for commuting costs described above, we can derive a simple expression for the elasticity of residential prices with respect to commuting costs.

Proposition 2: The elasticity of residential prices with respect to commuting costs, $\varepsilon_{q^{r}, \kappa}$, is given by $\kappa(B-|\ell|)$. 
Proof:

$$
\begin{gathered}
q^{r}(\ell)=\frac{e^{\kappa B}}{e^{\kappa|\ell|}} q_{B}^{r}=e^{\kappa(B-|\ell|)} q_{B}^{r} \\
\varepsilon_{q^{r}, \kappa}=\frac{\partial q^{r}(\ell)}{\partial \kappa} \cdot \frac{\kappa}{q^{r}(\ell)}=(B-|\ell|) e^{\kappa(B-|\ell|)} q_{B}^{r} \cdot \frac{\kappa}{e^{\kappa(B-|\ell|)} q_{B}^{r}}=\kappa(B-|\ell|) .
\end{gathered}
$$

The proposition above highlights the effect of commuting costs on prices; specifically, this effect is mitigated as we move away from the employment center and is zero at the boundary. Intuitively, away from the city center, residential prices become increasingly pinned down by the agricultural land rent rather than economic activity near the center.

Despite its richness, the stylized model we have just described imposes a number of restrictions on the structure of the city, including its monocentric nature with all production being concentrated in the CBD. Furthermore, residential prices decline monotonically as one moves away from the city center, and there exists a general symmetry and an evenness in allocations and prices across space. This smooth and symmetric aspect of the city is illustrated in Figure 1. In that figure, residential population is highest near the CBD, where the commute is relatively cheap, and decreases monotonically away from the center with the fewest workers living near the boundaries of the city.

In practice, of course, economic activity is more unevenly distributed across space. For example, Figure 2 shows that the city of Richmond, Virginia, has multiple employment clusters, one indeed in the center of the city but two others to the south and west.

This activity reflects a balance of agglomeration forces (e.g., production externalities) and dispersion forces (e.g., commuting costs) that play out in intricate and interrelated ways across space and that lead to substantial variations in allocations and prices across a city. For example, production may take place in different parts of the city so that cities with multiple production centers are not uncommon. In fact, some productive activity potentially takes place at every location in the city. Moreover, residential prices, even if they tend to fall away from a central point in the city, seldom fall monotonically with distance from that center. Instead, residential rents can exhibit substantial variation across locations within the city. This variation reflects the potential complexity of linkages within the city where, for example, the resident population at a given location may depend on the entire distribution of wages offered across the city. Thus, in the next section, we show how 
Waddell \& Sarte: From Stylized to Quantitative Spatial Models of Cities179

Figure 1 Allocations and Prices in the Monocentric Model

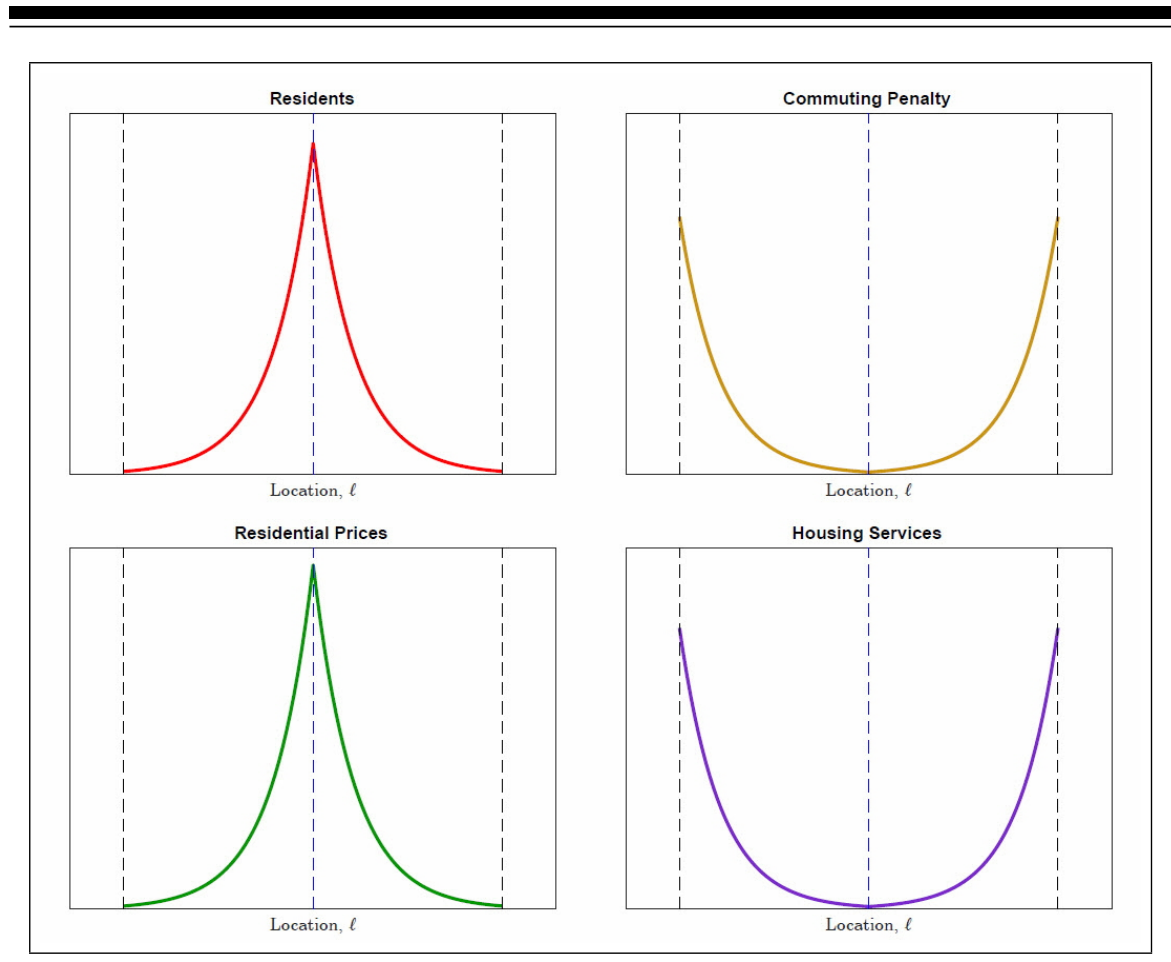

to modify the stylized model presented in this section in a way that can quantitatively account for the spatial allocations and prices it is meant to study.

\section{A QUANTITATIVE SPATIAL MODEL OF CITIES}

In this section, we show how to adapt the stylized model of the previous section to allow for the heterogeneity in spatial allocations and prices that is typically observed in cities. In doing so, we preserve the basic assumptions on preferences, technology, and endowments of our stylized model to keep the frameworks comparable. Instead of thinking of the city as located on an interval $[-B, B]$, we will think of the city as composed of $J$ distinct locations, indexed by $j \in\{1, \ldots, J\}$ (or $i$ ). In the mapping to data, these locations may represent city blocks, census tracts, or counties. It is this key change that will allow us to ensure that the model is at least able to match given observed spatial allocations of, for example, resident population, land rents, employment, or wages across locations in a city. Any subsequent counterfactual exer- 
Figure 2 Density of Primary Jobs

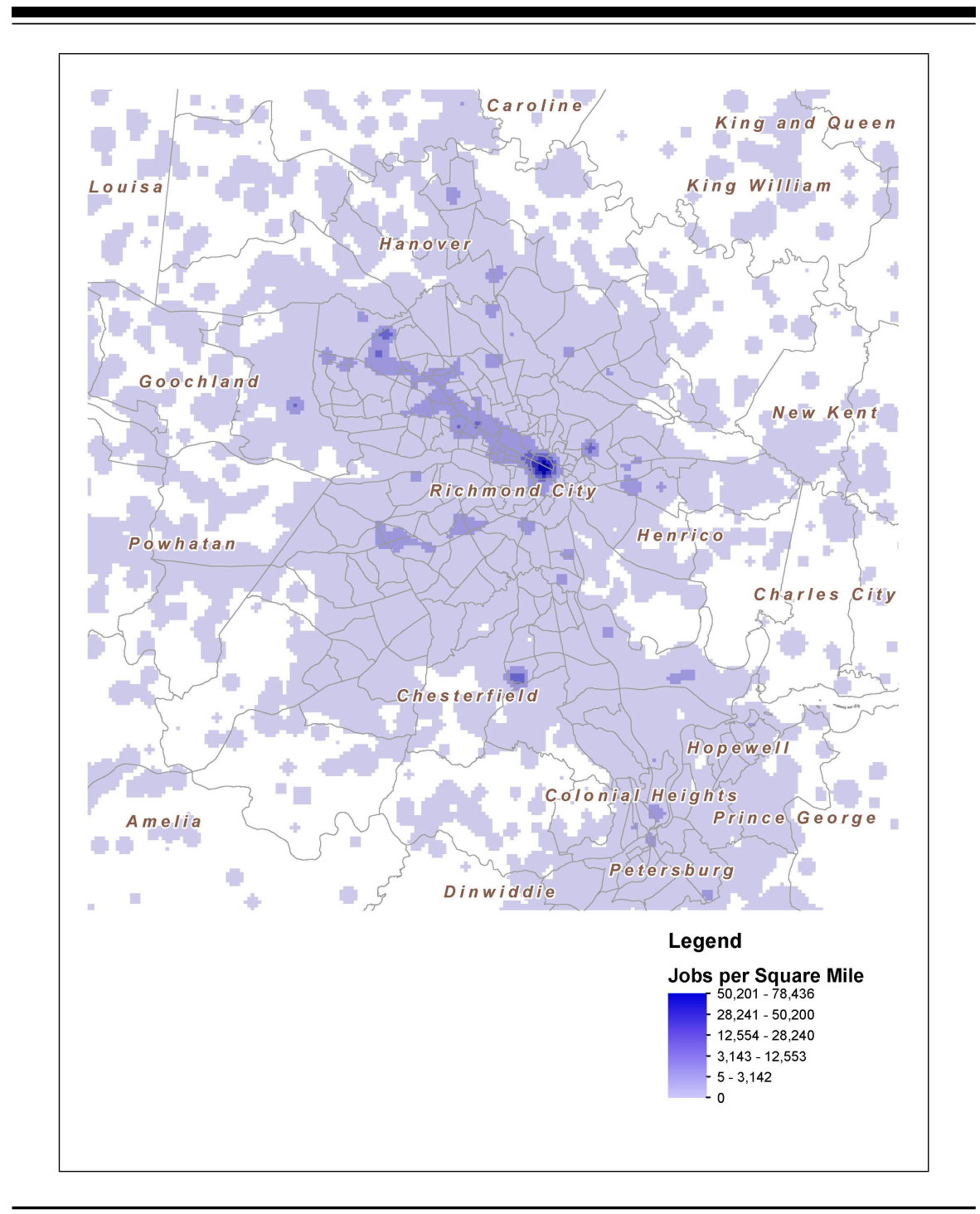

cise involving a change to some exogenous aspect of the city is then grounded in a model that is able to exactly replicate uneven spatial observations that reflect, at least in part, complex linkages between decisions involving where to reside and where to work within the city. For example, the model would enable us to understand the effect of a new urban policy, such as one that provides housing assistance or subsidized transportation. 
Waddell \& Sarte: From Stylized to Quantitative Spatial Models of Cities181

In a model where every location could potentially be used for both residential and production purposes, a central component of a quantitative spatial model is the representation and matching of distinct pairwise commuting flows from any location $j$ in the city to any other location $i$. This step will rely on an approach developed by Eaton and Kortum (2002) in modeling trade flows between locations. As in the model developed in the previous section, this analysis will focus on residential localization decisions, i.e., the decisions of households rather than firms. Unlike in the previous model, the commercial bid rent schedule is nondegenerate and reflects variations in productivity and wages across locations.

\section{Firms}

Production per unit of land in the business district of each location $i$ is given by

$$
Y_{i}=A\left(L_{i}\right) L_{i}^{\beta}
$$

analogously to equation (1), where $L_{i}$ denotes labor input and $A\left(L_{i}\right)$ denotes a production externality that we assume is local (so only employment in $i$ affects the productivity of businesses in $i$ ). For simplicity, let $A\left(L_{i}\right)=A_{i} L_{i}^{\alpha}, \alpha<1-\beta<1$, and denote the wage paid to workers in location $i$ by $w_{i}$. There exists a unit mass of firms (assuming that firms are small and do not internalize the externality) where the representative firm solves

$$
\max _{L_{i}} A\left(L_{i}\right) L_{i}^{\beta}-w_{i} L_{i} .
$$

It follows that

$$
\beta A\left(L_{i}\right) L_{i}^{\beta-1}=w \Leftrightarrow L_{i}=\left(\frac{A_{i} \beta}{w_{i}}\right)^{\frac{1}{1-\alpha-\beta}} .
$$

As in the previous model, firms operate in a competitive market with free entry and thus obtain zero profits in equilibrium. The implied commercial bid rent schedule faced by firms in the business district is

$$
q_{i}^{b}=(1-\beta) A_{i}^{\frac{1}{1-\alpha-\beta}}\left(\frac{\beta}{w_{i}}\right)^{\frac{\alpha+\beta}{1-\alpha-\beta}} .
$$

Note the similarities between equations (14) and (15), and the analogous equations in the previous section, equations (2) and (3). 


\section{Residents}

In each location $j$ of the city, there exists a residential area composed of a continuum of residents who commute to the business areas of different locations $i$ for work. These residents differ in their preferences for where to work in the city according to a random idiosyncratic component $s$. Unlike the previous model where $s$ was a city amenity distributed uniformly across locations, in this model, $s$ is an individual-specific preference component. Conditional on living in a particular location $j$, this preference component captures the idea that residents of $j$ may have idiosyncratic reasons for commuting to different locations $i$ in the city. We model the idiosyncratic preference component associated with residing in location $j$ and working in location $i$ as scaling the utility of the residents of region $j$, where $s$ is drawn from a Fréchet distribution specific to that particular commute,

$$
F_{i j}(s)=e^{-\lambda_{i j} s^{-\theta}}, \lambda_{i j}>0, \theta>0 .
$$

Residents of $j$ who commute to $i$ incur an associated cost, $\kappa_{i j} \in$ $[1, \infty)$, that, analogous to the previous section, reduces the utility derived from housing. Thus, conditional on living in $j$ and working in $i$, the problem of a resident having drawn idiosyncratic utility $s$ is given by

$$
\begin{aligned}
u_{i j}(s) & =\max _{c_{i j}(s), h_{i j}(s)} s\left(\frac{c_{i j}(s)}{\gamma_{j}}\right)^{\gamma_{j}}\left(\frac{h_{i j}(s)}{\left(1-\gamma_{j}\right) \kappa_{i j}}\right)^{1-\gamma_{j}}, \\
\gamma_{j} & \in(0,1)
\end{aligned}
$$

subject to $c_{i j}(s)+q_{j}^{r} h_{i j}(s)=w_{i}$

where $q_{j}^{r}$ is the price of a unit of residential housing services at location $j$. Hence, we have that

$$
\begin{aligned}
c_{i j}(s) & =\gamma_{j} w_{i}, \\
h_{i j}(s) & =\frac{\left(1-\gamma_{j}\right) w_{i}}{q_{j}^{r}},
\end{aligned}
$$

and

$$
\begin{aligned}
u_{i j}(s) & =s\left[w_{i}\right]^{\gamma_{j}}\left[\frac{w_{i}}{\kappa_{i j} q_{j}^{r}}\right]^{1-\gamma_{j}} \\
& =s w_{i}\left[\kappa_{i j} q_{j}^{r}\right]^{\gamma_{j}-1} .
\end{aligned}
$$

Note the similarities between equations (17), (18), and (19) and the analogous equations in the previous sections, equations (4), (5), and (6). 
Waddell \& Sarte: From Stylized to Quantitative Spatial Models of Cities183

\section{Aggregation}

The setup we have just described allows for a considerable degree of heterogeneity within the city compared to the stylized model presented earlier. In particular, all locations allow for simultaneous use by both businesses and residents (mixed use), individuals living in any location may commute to any other location for work, and commute costs between any two locations are specific to that pair of locations, so that it is possible to take into account the particular geographical makeup or road infrastructure of a city. However, having allowed for this high level of heterogeneity in the city, it becomes important to be able to aggregate economic activity at the level of a location, such as a census tract for practical purposes. The steps in this subsection address this question.

\section{Distribution of Utility}

Since residents of $j$ who work in $i$ have different preferences $s$, drawn from equation (16), for commuting to that location, it follows that

$$
G_{i j}(u)=\operatorname{Pr}\left(u_{i j}<u\right)=F_{i j}\left(\frac{u\left[\kappa_{i j} q_{j}^{r}\right]^{1-\gamma_{j}}}{w_{i}}\right),
$$

or

$$
G_{i j}(u)=e^{-\Phi_{i j} u^{-\theta}}, \Phi_{i j}=\lambda_{i j} w_{i}^{\theta}\left[\kappa_{i j} q_{j}^{r}\right]^{\left(\gamma_{j}-1\right) \theta} .
$$

Each resident of $j$ chooses to commute to the location $i$ that offers maximum utility of all possible locations. Therefore,

$$
\begin{aligned}
G_{j}(u) & =\operatorname{Pr}\left(\max _{i}\left\{u_{i j}\right\}<u\right)=\prod_{i} \operatorname{Pr}\left(u_{i j}<u\right) \\
& =\prod_{i} e^{-\Phi_{i j} u^{-\theta}} .
\end{aligned}
$$

Thus, it follows that

$$
G_{j}(u)=e^{-\Phi_{j} u^{-\theta}}, \Phi_{j}=\sum_{i} \Phi_{i j} .
$$

In other words, the distribution of resident utility in each location $j$ of the city is itself a Fréchet distribution. The expected utility from residing in $j$ is then given by

$$
u_{j}=\Gamma\left(\frac{\theta-1}{\theta}\right)\left(q_{j}^{r}\right)^{\gamma_{j}-1}\left(\sum_{i} \lambda_{i j} w_{i}^{\theta} \kappa_{i j}^{\left(\gamma_{j}-1\right) \theta}\right)^{\frac{1}{\theta}},
$$


where $\Gamma($.$) is the Gamma function. { }^{1}$ The expected utility from living in $j$, therefore, is a weighted average of the utility gained from commuting to the different business areas (raised to the $\theta$ ). Observe that in contrast to utility in the stylized model, equation (6), the expected utility from living in location $j$ of the city now involves not only the price of housing at that location, but also information about the entire city, including the entire distribution of wages and associated commuting costs, since residents of $j$ can in principle commute to any other location $i$ to work.

\section{Commuting Patterns}

Let $\pi_{i j}$ represent the proportion of residents living at location $j$ and commuting to location $i$. Commuting patterns can then be described by the following relationship,

$$
R_{i j}=\pi_{i j} R_{j},
$$

where $R_{i j}$ and $R_{j}$ are, respectively, the number of residents commuting from $j$ to $i$ and the total number of residents living at $j$. In particular,

$$
\pi_{i j}=\operatorname{Pr}\left[u_{i j}>\max _{n \neq i}\left\{u_{n j}\right\}\right] .
$$

From equation (20), we have that $G_{i j}(u)=e^{-\Phi_{i j} u^{-\theta}}$ so that $g_{i j}(u)=$ $\theta u^{-(\theta+1)} \Phi_{i j} e^{-\Phi_{i j} u^{-\theta}}$. It follows that

$$
\pi_{i j}=\int_{0}^{\infty} \theta u^{-(\theta+1)} \Phi_{i j} e^{-\Phi_{i j} u^{-\theta}} \widetilde{G}_{j}(u) d u,
$$

where $\widetilde{G}_{j}(u)$ is defined as in equation (21) but with $\widetilde{\Phi}_{j}=\sum_{n \neq j} \Phi_{n j}$, which also implies that $\Phi_{j}=\widetilde{\Phi}_{j}+\Phi_{i j}$. In Appendix B, we show that this expression reduces to

$$
\pi_{i j}=\frac{\lambda_{i j} w_{i}^{\theta} \kappa_{i j}^{\left(\gamma_{j}-1\right) \theta}}{\sum_{i} \lambda_{i j} w_{i}^{\theta} \kappa_{i j}^{\left(\gamma_{j}-1\right) \theta}}
$$

In other words, the proportion of residents living in $j$ and commuting to $i$ for work depends on wages earned in $i$ adjusted for commuting costs when coming from $j$, relative to a weighted average of wages earned elsewhere adjusted for the corresponding commute (raised to the $\theta$ ).

\footnotetext{
${ }^{1} \mathrm{~A}$ derivation of this result is given in Appendix A.
} 
Waddell \& Sarte: From Stylized to Quantitative Spatial Models of Cities185

The residential price at location $j$ does not affect commuting patterns from $j$ to $i$ since it is specific to $j$ and is faced by any resident who wants to live at $j$ regardless of commute. By construction, $\sum_{i} \pi_{i j}=1$.

\section{The Residential Market}

Recall that $h_{i j}(s)=h_{i j}$ represents housing consumption for those living in $j$ and commuting to $i$. It follows that average housing per resident at location $j, h_{j}$, is given by

$$
\begin{aligned}
h_{j} & =\sum_{i} \pi_{i j} h_{i j} \\
& =\frac{\left(1-\gamma_{j}\right)}{q_{j}^{r}} \sum_{i} \pi_{i j} w_{i} .
\end{aligned}
$$

As in the stylized model of the previous section, we assume that each location is endowed with one unit of land available for housing and that this land is fully developed. ${ }^{2}$ In equilibrium, therefore, the residential market must satisfy $R_{j} h_{j}=1$ similarly to equation (9) or

$$
R_{j}=\frac{q_{j}^{r}}{\left(1-\gamma_{j}\right) \sum_{i} \pi_{i j} w_{i}} .
$$

As in the previous section, let $\bar{u}$ denote the utility available to individuals from residing in alternative cities. To the extent that workers can move to or from another city, and are free to reside at any location within the city, it must be the case that in equilibrium $u_{j}=\bar{u} \forall j$. Therefore, we have that for any location,

$$
\begin{aligned}
\bar{u} & =\Gamma\left(\frac{\theta-1}{\theta}\right)\left(q_{j}^{r}\right)^{\gamma_{j}-1}\left(\sum_{i} \lambda_{i j} w_{i}^{\theta} \kappa_{i j}^{\left(\gamma_{j}-1\right) \theta}\right)^{\frac{1}{\theta}} \\
& \Rightarrow q_{j}^{r}=\left[\frac{\bar{u}}{\Gamma\left(\frac{\theta-1}{\theta}\right)}\right]^{\frac{1}{\gamma_{j}-1}}\left(\sum_{i} \lambda_{i j} w_{i}^{\theta} \kappa_{i j}^{\left(\gamma_{j}-1\right) \theta}\right)^{\frac{1}{\theta\left(1-\gamma_{j}\right)}} .
\end{aligned}
$$

Comparing the residential price at location $j, q_{j}^{r}$, with its simpler analog in the stylized model in equation (8), it is clear that the quantitative spatial model allows residential prices to be determined by many more

\footnotetext{
${ }^{2}$ Owens et al. (2017) present a more flexible model in which residential land in any one location may be vacant, partially developed with some areas left for developers to build on, or fully developed. In that model, a coordination problem arises between developers and residents (no one wants to be the first mover) that potentially traps neighborhoods in an equilibrium where they remain vacant.
} 
factors, including the distribution of wages across all locations in the city as well as all commuting costs. It is this richness that allows for spatial variation in allocations and prices across locations in the city that is unavailable in the more stylized framework of the previous section.

\section{The City Labor Market}

Since $\pi_{i j} R_{j}$ denotes the number of residents living in location $j$ who commute to the business area of location $i$ for work, labor market clearing in the city requires that

$$
L_{i}=\sum_{j=1}^{J} \pi_{i j} R_{j},
$$

or alternatively

$$
\left(\frac{A_{i} \beta}{w_{i}}\right)^{\frac{1}{1-\alpha-\beta}}=\sum_{j=1}^{J} \pi_{i j} R_{j} .
$$

\section{Solving for the City Equilibrium}

We represent the parameters of the quantitative spatial model in a vector, $\mathcal{P}=\left(\alpha, \beta, \theta, \bar{u}, \gamma_{j}, A_{i}, \kappa_{i j}, \lambda_{i j}\right)$. Conditional on $\mathcal{P}$, equations (24), (25), (26), and (27) then make up a system of $J^{2}+3 J$ equations in the same number of unknowns, $\pi_{i j}(\mathcal{P}), R_{j}(\mathcal{P}), q_{j}^{r}(\mathcal{P})$, and $w_{i}(\mathcal{P})$.

Importantly, the equilibrium allocations in this model allow for considerably more heterogeneity than in the stylized model of the previous section. Since they are specific to locations within the city, equilibrium allocations of the quantitative spatial model such as commuting patterns, $\pi_{i j}(\mathcal{P})$, or equilibrium prices, such as residential prices, $q_{j}^{r}(\mathcal{P})$, and wages, $w_{i}(\mathcal{P})$, may be directly matched to their data counterpart at the block or census tract level. In contrast, equilibrium allocations of the stylized model in the previous section could only be indexed by distance, $\ell$, from a central point in the city. The next section addresses this last point in more detail.

Unlike the conventional monocentric model of the previous section, equilibrium existence and uniqueness are more challenging to prove in a quantitative spatial framework. However, Appendix C summarizes the key equations needed to compute the model equilibrium and provides an algorithm that yields the corresponding numerical solution given the model's parameters, $\mathcal{P}$. Moreover, despite its added complexity, the quantitative spatial model retains some degree of analytical tractability. 
Waddell \& Sarte: From Stylized to Quantitative Spatial Models of Cities187

For instance, as in the monocentric model of the previous section, we can derive a simple expression for the elasticity of residential prices with respect to commuting costs.

Proposition 3: The elasticity of residential prices with respect to commuting costs, $\varepsilon_{q_{j}^{r}, \kappa_{i j}}$, is given by $-\pi_{i j}$.

Proof: We have that $q_{j}^{r}=\left(\frac{\bar{u}}{\Gamma\left(\frac{\theta-1}{\theta}\right)}\right)^{\frac{1}{\gamma_{j}-1}}\left(\sum_{i} \lambda_{i j} w_{i}^{\theta} \kappa_{i j}^{\left(\gamma_{j}-1\right) \theta}\right)^{\frac{1}{\theta\left(1-\gamma_{j}\right)}}$. Then

$$
\begin{gathered}
\frac{\partial q_{j}^{r}}{\partial \kappa_{i j}}= \\
\left(\frac{\bar{u}}{\Gamma\left(\frac{\theta-1}{\theta}\right)}\right)^{\frac{1}{\gamma_{j}-1}} \frac{1}{\theta\left(1-\gamma_{j}\right)}\left(\sum_{i} \lambda_{i j} w_{i}^{\theta} \kappa_{i j}^{\left(\gamma_{j}-1\right) \theta}\right)^{\frac{1}{\theta\left(1-\gamma_{j}\right)}-1}\left(\gamma_{j}-1\right) \theta \lambda_{i j} w_{i}^{\theta} \kappa_{i j}^{\left(\gamma_{j}-1\right) \theta-1} \\
=-1 \cdot \frac{\lambda_{i j} w_{i}^{\theta} \kappa_{i j}^{\left(\gamma_{j}-1\right) \theta-1}}{\sum_{i} \lambda_{i j} w_{i}^{\theta} \kappa_{i j}^{\left(\gamma_{j}-1\right) \theta}} q_{j}^{r}
\end{gathered}
$$

It follows that

$$
\varepsilon_{q_{j}^{r}, \kappa_{i j}}=\frac{\partial q_{j}^{r}}{\partial \kappa_{i j}} \cdot \frac{\kappa_{i j}}{q_{j}^{r}}=-\frac{\lambda_{i j} w_{i}^{\theta} \kappa_{i j}^{\left(\gamma_{j}-1\right) \theta-1} q_{j}^{r}}{\sum_{i} \lambda_{i j} w_{i}^{\theta} \kappa_{i j}^{\left(\gamma_{j}-1\right) \theta}} \cdot \frac{\kappa_{i j}}{q_{j}^{r}}=-\pi_{i j} .
$$

This finding is intuitive. A 1 percent increase in commute costs between any two locations, $\kappa_{i j}$, lowers residential prices by the share of residents affected by that commute. In this relatively simple spatial environment, even if it allows for more flexibility than the monocentric setup, the relationship is exact. More importantly, unlike the analogous elasticity in the more stylized model, the share of residents is itself an endogenous outcome that depends on all of the city's characteristics, $\mathcal{P}$, and thus will move along with the entire distribution of wages and population across locations in any policy experiment.

\section{MATCHING THE QUANTITATIVE SPATIAL MODEL TO URBAN MICRODATA}

As elaborated upon in earlier sections, it is now possible to model cities by matching these types of quantitative spatial models to available microdata. For the purpose of the discussion below, the parameters in $\mathcal{P}$ fall into two broad classifications: citywide parameters and locationor neighborhood-specific parameters. The parameters in $\mathcal{P}$ that are 
not location specific have generally accepted values in the literature. For example, Monte et al. (2016) estimate $\theta$ (the parameter that governs the shape of the distribution of the idiosyncratic preference, $s$, of commuting from $i$ to $j$ ) to be 4.43. Ciccone and Hall (1996) estimate $\alpha$ (the production externality) to be 0.06, and Ahlfeldt et al. (2015) estimate $\beta$ (the parameter that defines the relationship between labor and output, separate from the externality) to be 0.80 , while $\bar{u}$ is a normalizing constant. The parameters that are location-specific potentially present a greater computational challenge since there are many of them. For example, in a city with 1,000 census tracts, there are $1,000,000 \lambda_{i j}$ 's. Other location-specific parameters, such as pairwise commuting costs, $\kappa_{i j}$, may be directly calibrated to data on distances or commuting times.

The Longitudinal Employer-Household Dynamics Origin-Destination Employment Statistics provide reliable data on cities at the census tract level, including commuting patterns $\left(\pi_{i j}\right)$, resident population $\left(R_{j}\right)$, employment $\left(L_{i}\right)$, and wages $\left(w_{i}\right)$. Other detailed data on cities are also available; for example, residential prices $\left(q_{j}^{r}\right)$ are available from CoreLogic or county assessors' offices. In general, such data show considerable unevenness across space within a city.

We now describe how, in our simple framework, the location-specific parameters of our quantitative spatial model may be calibrated to exactly match, in equilibrium, all pairwise commuting patterns, $\pi_{i j}$, the exact distribution of population across space, $R_{j}$, and thus also the distribution of employment, $L_{i}$, and the exact distribution of wages in the city, $w_{i}$, in a given benchmark period. In particular, we choose the parameters of the model $\left(\lambda_{i j}, \gamma_{j}, A_{i}\right)$ to match the observations $\left(\pi_{i j}, R_{j}, w_{i}\right)$ as equilibrium outcomes. In this way, counterfactual exercises involving a change to some exogenous aspect of the city, or a change in urban policy, are rooted in a model that, as a benchmark, is able to exactly match basic observed allocations and prices in the city as equilibrium outcomes.

Recall that commuting patterns, $\pi_{i j}$, are given by equation (24),

$$
\pi_{i j}=\frac{\lambda_{i j} w_{i}^{\theta} \kappa_{i j}^{\left(\gamma_{j}-1\right) \theta}}{\sum_{i} \lambda_{i j} w_{i}^{\theta} \kappa_{i j}^{\left(\gamma_{j}-1\right) \theta}},
$$

where $\kappa_{i j} \in[1, \infty)$. If $\pi_{i j}=0$, then either $\lambda_{i j}=0$ or $\kappa_{i j} \rightarrow \infty$. Commuting patterns can be alternatively expressed in terms of the Head and Ries (2001) index,

$$
\frac{\pi_{i j}}{\pi_{j j}}=\frac{\lambda_{i j} w_{i}^{\theta} \kappa_{i j}^{\left(\gamma_{j}-1\right) \theta}}{\lambda_{j j} w_{j}^{\theta} \kappa_{j j}^{\left(\gamma_{j}-1\right) \theta}} .
$$


Waddell \& Sarte: From Stylized to Quantitative Spatial Models of Cities189

Then, conditional on $\theta=4.43$ and values for $\gamma_{j}$, the preference parameters, $\lambda_{i j}$, can then be chosen to be consistent with commuting patterns,

$$
\lambda_{i j}=\pi_{i j}\left(\frac{w_{j}}{w_{i}}\right)^{\theta}\left(\frac{\kappa_{i j}}{\kappa_{\min }}\right)^{\left(1-\gamma_{j}\right) \theta}\left(\frac{\lambda_{j j}}{\pi_{j j}}\right),
$$

where $\kappa_{\text {min }}$ is a lower bound on $\kappa_{j j} .{ }^{3}$ Since $\kappa_{i j}$ may be directly inferred from commuting costs data, this approach to obtaining $\lambda_{i j}$ to match commuting patterns presumes we are also able to match wages, $w_{i}$, as part of the model inversion. We show below that this can indeed be done through the choice of location-specific productivities, $A_{i}$. With this in mind, we first choose $\gamma_{j}$ so as to match the distribution of resident population across space, $R_{j}$, conditional on $\pi_{i j}$ and $w_{i}$.

The number of residents in location $j$ is given by

$$
R_{j}=\frac{q_{j}^{r}}{\left(1-\gamma_{j}\right) \sum_{i} \pi_{i j} w_{i}} .
$$

Using equations (26) and (28) with $\kappa_{\min }=1$, and the normalization $\left(\frac{\lambda_{j j}}{\pi_{j j}}\right)=1$, for all locations $j$, equation (29) simplifies to

$$
R_{j}\left(\gamma_{j}\right)=\left(\frac{\Gamma\left(\frac{\theta-1}{\theta}\right) w_{j}}{\bar{u}}\right)^{\frac{1}{1-\gamma_{j}}} \frac{1}{\left(1-\gamma_{j}\right) \sum_{i} \pi_{i j} w_{i}} .
$$

Notice that as $\gamma_{j} \rightarrow 0^{+}, R_{j}\left(\gamma_{j}\right) \rightarrow \frac{\Gamma\left(\frac{\theta-1}{\theta}\right) w_{j}}{\bar{u} \sum_{i} \pi_{i j} w_{i}}$ and as $\gamma_{j} \rightarrow 1^{-}, R_{j}\left(\gamma_{j}\right) \rightarrow$ $\infty$. Therefore, one may choose $\bar{u}$ so that $R_{j}>\frac{\Gamma\left(\frac{\theta-1}{\theta}\right) w_{j}}{\bar{u} \sum_{i} \pi_{i j} w_{i}}$ for all $j$ and numerically solve the expression in equation (30) to obtain a set of $\gamma_{j}$ that exactly matches the distribution of $R_{j}$, conditional on $\pi_{i j}$ and $w_{i}$. Since the distribution of $\gamma_{j}$ then depends on $\bar{u}$, and $\gamma_{j}$ represents the share of income spent on housing in a given census tract $j$, we choose $\bar{u}$ so that the mean of $\gamma_{j}$ is 0.76 as in Ahlfeldt et al. (2015).

Since commuting patterns $\pi_{i j}$ can be exactly matched, given $\gamma_{j}$ and wages $w_{i}$, through the choice of $\lambda_{i j}$ in equation (28), it remains only to ensure that the model is consistent with the spatial distribution of wages in an equilibrium benchmark version of the model. Using equation (26), we can write the city labor market clearing condition, equation (27) as

$$
\left(\frac{A_{i} \beta}{w_{i}}\right)^{\frac{1}{1-\alpha-\beta}}=\sum_{j=1}^{J} \pi_{i j} R_{j}
$$

\footnotetext{
${ }^{3}$ Since $\sum_{i} \pi_{i j}=1$, one needs to also normalize the $\lambda_{i j}$ 's, for example, $\frac{\lambda_{j j}}{\kappa_{j j}}=1 \forall j$.
} 
in which case we can simply choose location-specific productivities, $A_{i}$, to ensure that equilibrium benchmark wages exactly match the distribution of wages in the city,

$$
A_{i}=\frac{w_{i}}{\beta}\left\{\sum_{j=1}^{J} \pi_{i j} R_{j}\right\}^{1-\alpha-\beta}
$$

Observe that on the righthand side of equation (31), we are free to use the data on commuting patterns, $\pi_{i j}$, and residential population, $R_{j}$, since those are matched by construction through the choices of $\lambda_{i j}$ and $\gamma_{j}$ in equations (28) and (30).

\section{CONCLUSION}

The development of the new quantitative equilibrium models has initiated a more robust and realistic framework with which to model cities. This framework will enable urban economists to provide empirically driven insight into future theoretical or structural work on how cities grow, shrink, and change. By offering a more accurate grounding for empirical models, it will also allow for more robust counterfactual policy exercises that can inform practitioners and policymakers regarding strategies for urban development. 
Waddell \& Sarte: From Stylized to Quantitative Spatial Models of Cities191

\section{REFERENCES}

Ahlfeldt, Gabriel M., Stephen J. Redding, Daniel M. Sturm, and Nikolaus Wolf. 2015. "The Economics of Density: Evidence from the Berlin Wall." Econometrica 83 (November): 2127-89.

Ciccone, Antonio, and Robert E. Hall. 1996. "Productivity and the Density of Economic Activity." American Economic Review 86 (March): 54-70.

Eaton, Jonathan, and Samuel Kortum. 2002. "Technology, Geography, and Trade." Econometrica 70 (September): 1741-79.

Fujita, Masahisa. 1988. "A Monopolistic Competition Model of Spatial Agglomeration: Differentiated Product Approach." Regional Science and Urban Economics 18 (February): 87-124.

Fujita, Masahisa, Paul Krugman, and Anthony J. Venables. 1999. The Spatial Economy: Cities, Regions, and International Trade. Cambridge, Mass.: MIT Press.

Fujita, Masahisa, and Hideaki Ogawa. 1982. "Multiple Equilibria and Structural Transition of Non-Monocentric Urban Configurations." Regional Science and Urban Economics 12 (May): 161-96.

Fujita, Masahisa, and Jacques-François Thisse. 2002. Economics of Agglomeration: Cities, Industrial Location, and Regional Growth. Cambridge: Cambridge University Press.

Krugman, Paul. 1991. "Increasing Returns and Economic Geography." Journal of Political Economy 99 (June): 483-99.

Lucas, Robert E., and Esteban Rossi-Hansberg. 2002. "On the Internal Structure of Cities." Econometrica 70 (July): 1445-76.

Monte, Ferdinando, Stephen J. Redding, and Esteban Rossi-Hansberg. 2016. "Commuting, Migration, and Local Employment Elasticities." Princeton University Working Paper (October).

Ottaviano, Gianmarco, and Jacques-François Thisse. 2004. "Agglomeration and Economic Geography." In Handbook of Regional and Urban Economics, vol. 4, ed. J. Vernon Henderson and Jacques-François Thisse. North Holland: Elsevier: 2563-608.

Owens, Raymond III, Esteban Rossi-Hansberg, and Pierre-Daniel Sarte. 2017. "Rethinking Detroit." Working Paper 23146. Cambridge, Mass.: National Bureau of Economic Research. (February). 
Redding, Stephen J., and Esteban Rossi-Hansberg. Forthcoming. "Quantitative Spatial Economics." Annual Review of Economics.

Redding, Stephen J., and Daniel M. Sturm. 2008. "The Costs of Remoteness: Evidence from German Division and Reunification." American Economic Review 98 (December): 1766-97.

Venables, Anthony J. 1996. "Equilibrium Locations of Vertically Linked Industries." International Economic Review 37 (May): $341-59$. 
Waddell \& Sarte: From Stylized to Quantitative Spatial Models of Cities193

\section{APPENDIX: APPENDIX A}

Under the maintained assumptions, expected utility at location $j$ is given by

$$
u_{j}=\int_{0}^{\infty} \theta \Phi_{j} u^{-\theta} e^{-\Phi_{j} u^{-\theta}} d u
$$

Consider the change in variables,

$$
y=\Phi_{j} u^{-\theta}, d y=-\theta \Phi_{j} u^{-(\theta+1)} d u .
$$

Then, we have that

$$
u_{j}=\int_{0}^{\infty} \Phi_{j}^{\frac{1}{\theta}} y^{\frac{-1}{\theta}} e^{-y} d y=\Gamma\left(\frac{\theta-1}{\theta}\right) \Phi_{j}^{\frac{1}{\theta}}
$$

from which equation (22) in the text follows.

\section{APPENDIX: APPENDIX B}

From equation (23), we have that

$$
\begin{aligned}
\pi_{i j} & =\int_{0}^{\infty} \theta u^{-(\theta+1)} \Phi_{i j} e^{-\Phi_{i j} u^{-\theta}} e^{-\widetilde{\Phi}_{j} u^{-\theta}} d u \\
& =\int_{0}^{\infty} \theta u^{-(\theta+1)} \Phi_{i j} e^{-\Phi_{j} u^{-\theta}} d u .
\end{aligned}
$$

Consider the change of variables,

$$
y=\Phi_{j} u^{-\theta}, d y=-\theta \Phi_{j} u^{-(\theta+1)} d u .
$$


It then follows that

$$
\begin{aligned}
\pi_{i j} & =\Phi_{i j} \int_{0}^{\infty} \theta u^{-(\theta+1)} e^{-\Phi_{j} u^{-\theta}} d u \\
& =\frac{\Phi_{i j}}{\Phi_{j}} \int_{0}^{\infty} e^{-y} d y \\
& =\frac{\Phi_{i j}}{\Phi_{j}}
\end{aligned}
$$

where recall that $\Phi_{i j}=\lambda_{i j} w_{i}^{\theta}\left[\kappa_{i j} q_{j}^{r}\right]^{\left(\gamma_{j}-1\right) \theta}$ and $\Phi_{j}=\left(q_{j}^{r}\right)^{\left(\gamma_{j}-1\right) \theta} \sum_{i} \lambda_{i j} w_{i}^{\theta} \kappa_{i j}^{\left(\gamma_{j}-1\right) \theta}$. Equation (24) in the text directly follows.

\section{APPENDIX: APPENDIX C}

\section{The Basic Set of Equations and Unknowns}

Let $L_{i}^{D}$ and $L_{i}^{S}$ represent, respectively, labor demand and labor supply in location $i$. Given a benchmark or counterfactual set of parameters, $\mathcal{P}$, each endogenous variable in the model can ultimately be expressed as depending only on a vector of wages across all locations, $\boldsymbol{w}=\left(w_{1}, \ldots, w_{J}\right)^{\prime}$, and $\mathcal{P}$,

$$
\begin{aligned}
\pi_{i j}(\boldsymbol{w}) & =\frac{\lambda_{i j} w_{i}^{\theta} \kappa_{i j}^{\left(\gamma_{j}-1\right) \theta}}{\sum_{i} \lambda_{i j} w_{i}^{\theta} \kappa_{i j}^{\left(\gamma_{j}-1\right) \theta}} \\
q_{j}^{r}(\boldsymbol{w}) & =\left[\frac{\bar{u}}{\Gamma\left(\frac{\theta-1}{\theta}\right)}\right]^{\frac{1}{\gamma_{j}-1}}\left(\sum_{i} \lambda_{i j} w_{i}^{\theta} \kappa_{i j}^{\left(\gamma_{j}-1\right) \theta}\right)^{\frac{1}{\theta\left(1-\gamma_{j}\right)}}, \\
R_{j}(\boldsymbol{w}, \boldsymbol{\pi}, \boldsymbol{q}) & =\frac{q_{j}^{r}}{\left(1-\gamma_{j}\right) \sum_{i} \pi_{i j} w_{i}}, \\
L_{i}^{D}(\boldsymbol{w}) & =\left(\frac{A_{i} \beta}{w_{i}}\right)^{\frac{1}{1-\alpha-\beta}}, \\
L_{i}^{S}(\boldsymbol{\pi}, \boldsymbol{R}) & =\sum_{j} \pi_{i j} R_{j} .
\end{aligned}
$$


Waddell \& Sarte: From Stylized to Quantitative Spatial Models of Cities195

Then, finding an equilibrium of the model is equivalent to finding a vector of wages that clears the labor market; $L_{i}^{D}(\boldsymbol{w})=L_{i}^{S}(\boldsymbol{w})$ for all locations $i=1, \ldots, J$. Put another way, the task is to find a vector $\boldsymbol{w}^{*} \in \mathbb{R}_{+}^{J}$ such that

$$
\begin{gathered}
\left(L_{i}^{D}-L_{i}^{S}\right)\left(\boldsymbol{w}^{*}\right)= \\
\left(\frac{A_{i} \beta}{w_{i}}\right)^{\frac{1}{1-\alpha-\beta}}-\sum_{j} \frac{\lambda_{i j} w_{i}^{\theta} \kappa_{i j}^{\left(\gamma_{j}-1\right) \theta}}{\sum_{i} \lambda_{i j} w_{i}^{\theta} \kappa_{i j}^{\left(\gamma_{j}-1\right) \theta}} \frac{\left[\frac{\bar{u}}{\Gamma\left(\frac{\theta-1}{\theta}\right)}\right]^{\frac{1}{\gamma_{j}-1}}\left(\sum_{i} \lambda_{i j} w_{i}^{\theta} \kappa_{i j}^{\left(\gamma_{j}-1\right) \theta}\right)^{\frac{1}{\theta\left(1-\gamma_{j}\right)}}}{\left(1-\gamma_{j}\right) \sum_{i} \frac{\lambda_{i j} w_{i}^{\theta} \kappa_{i j}^{\left(\gamma_{j}-1\right) \theta}}{\sum_{i} \lambda_{i j} w_{i}^{\theta} \kappa_{i j}^{\left(\gamma_{j}-1\right) \theta}} w_{i}} \\
=\mathbf{0}
\end{gathered}
$$

Several algorithms exist to numerically solve nonlinear system of equations, and MATLAB's fsolve function handles this particular system well.

\section{Numerical Algorithm}

Some quantitative spatial models can result in systems whose features (such as nondifferentiability in the presence of thresholds or binding constraints on available land) make traditional algorithms difficult to apply. In such cases, a simple "guess-and-iterate" method can be constructed to calculate solutions. We outline such a method here as it applies to our model.

1. Choose a tolerance level $\varepsilon>0$ and guess a vector of wages, $\boldsymbol{w}_{n}$.

2. Calculate the implied matrix of flows: $\pi_{i j}\left(\boldsymbol{w}_{n}\right)=\frac{\lambda_{i j} w_{n, i}^{\theta} \kappa_{i j}^{\left(\gamma_{j}-1\right) \theta}}{\sum_{i} \lambda_{i j} w_{n, i}^{\theta} \kappa_{i j}^{\left(\gamma_{j}-1\right) \theta}}$.

3. Calculate the implied prices:

$$
q_{j}^{r}\left(\boldsymbol{w}_{n}\right)=\left[\frac{\bar{u}}{\Gamma\left(\frac{\theta-1}{\theta}\right)}\right]^{\frac{1}{\gamma_{j}-1}}\left(\sum_{i} \lambda_{i j} w_{n, i}^{\theta} \kappa_{i j}^{\left(\gamma_{j}-1\right) \theta}\right)^{\frac{1}{\theta\left(1-\gamma_{j}\right)}} .
$$

4. Using the prices and flows calculated in steps two and three, calculate the implied number of residents: $R_{j}\left(\boldsymbol{w}_{n}\right)=\frac{q_{j}^{r}\left(\boldsymbol{w}_{n}\right)}{\left(1-\gamma_{j}\right) \sum_{i} \pi_{i j}\left(\boldsymbol{w}_{n}\right) w_{i}}$.

5. Using the residents calculated in step four, calculate the implied labor supply in each labor market: $L_{i}^{S}\left(\boldsymbol{w}_{n}\right)=\sum_{j} \pi_{i j}\left(\boldsymbol{w}_{n}\right) R_{j}\left(\boldsymbol{w}_{n}\right)$.

6. Calculate the implied labor demand in each labor market: $L_{i}^{D}\left(\boldsymbol{w}_{n}\right)=$ $\left(\frac{A_{i} \beta}{w_{i}}\right)^{\frac{1}{1-\alpha-\beta}}$. 
7. At the vector of wages, $\boldsymbol{w}_{n}$, calculate the implied excess demand for labor in each market: $L_{i}^{D}\left(\boldsymbol{w}_{n}\right)-L_{i}^{S}\left(\boldsymbol{w}_{n}\right)$.

8. If the aggregate labor market fails to clear, $\sum_{i}\left|L_{i}^{D}\left(\boldsymbol{w}_{n}\right)-L_{i}^{S}\left(\boldsymbol{w}_{n}\right)\right|>$ $\varepsilon$, then update the vector of wages as follows:

$$
\boldsymbol{w}_{n+1}=\boldsymbol{w}_{n}+\delta\left(L_{i}^{D}\left(\boldsymbol{w}_{n}\right)-L_{i}^{S}\left(\boldsymbol{w}_{n}\right)\right),
$$

for some $\delta>0$. This updating rule raises wages in markets where there is excess demand for labor or reduces it where there is excess supply.

9. Repeat steps two through eight until $\sum_{i}\left|L_{i}^{D}\left(\boldsymbol{w}_{n}\right)-L_{i}^{S}\left(\boldsymbol{w}_{n}\right)\right| \leq \varepsilon$. 